\title{
Some New Surfaces of General Type
}

\author{
Masahisa INOUE \\ Aoyamagakuin University \\ (Communicated by T. Nagano)
}

\section{Introduction.}

In this paper we shall give two series of rare examples of algebraic surfaces of general type. One is a series of surfaces with positive topological indices and another with the geometric genus $p_{g}=0$.

In Part I, we construct surfaces with positive topological indices. In [V] (1966), A. Van de Ven pointed out that there are not many known examples of algebraic surfaces of general type with positive topological indices. By the index theorem of Hirzebruch, the topological index $\tau(S)$ of a surface $S$ is equal to $\left(K_{S}^{2}-2 e(S)\right) / 3$ where $K_{S}$ is the canonical line bundle and $e(S)$ the topological Euler number. Hence the positivity of $\tau(S)$ is equivalent to $K_{S}^{2}>2 e(S)$. At that time, the only known examples were the ones due to $F$. Hirzebruch $[\mathrm{H}]$, which are the compact quotients of the 2-dimensional unit ball and which therefore satisfy $K_{S}^{2}=3 e(S)$. Following Van de Ven's remark, K. Kodaira [K] (1967) was the first to construct a series of examples as branched coverings of the product of two algebraic curves. Kodaira's examples satisfy $3 e(s)>K_{S}^{2}>2 e(S)$ and have many interesting properties. Afterwards some new examples were discovered, for instance, by Mostow-Siu (1979) and Y. Miyaoka (1980). But even now the known examples are rare. In Part I, we construct new examples of such surfaces as branched coverings of the so-called elliptic modular surfaces which are investigated in detail in T. Shioda [S] (1972). Our construction depends heavily on some results in [S], which we shall recall in $\S 1$. We remark that our examples are discovered independently also by $\mathbf{R}$. Livné.

In Part II, we construct surfaces with $p_{g}=0$. If $S$ is a minimal surface of general type with $p_{g}(S)=0$, then we know generally that $q(S)=0, K_{S}^{2}=1,2, \cdots, 9$. Till 1974, the known and verified example was only the classical one due to L. Godeaux, on which we refer to Y. Miyaoka [M] (1976). Afterwards some classically known examples were verified and furthermore some new examples were discovered by, for instance, R. Barlow, A. Beauville, Y. Miyaoka, D. Mumford, C. A. M. Peters, M. Reid and I. Shavel and 
so on. But even now the known examples are rare. In Part II, we construct new examples of such surfaces as the quotients of the hypersurfaces of the product of three elliptic curves. Our construction is very elementary and has a close relation with a classical example due to P. Burniat, a geometer of the classical Italian school (see $\left[\mathrm{I}_{2}\right]$ ).

The main part of this work was done during the author's stay at Bonn University from September 1979 to August 1980. The author would like to express his sincere thanks to Professor F. Hirzebruch, who provided him the opportunity to stay in Bonn, as well as warm encouragement and many invaluable suggestions.

\section{PART I Surfaces of General Type with Positive Topological Indices}

\section{§1. Elliptic modular surfaces (due to T. Shioda).}

In this section, we shall recall some results in T. Shioda [S]. From now on, we assume that $N \geqq 3$. Let $\Gamma(N)$ be the principal congruence subgroup of level $N$, namely,

$$
\Gamma(N)=\left\{\left(\begin{array}{ll}
a & b \\
c & d
\end{array}\right) \in \mathrm{SL}(2, Z) \mid a, d \equiv 1, b, c \equiv 0 \text { modulo } N\right\} .
$$

Let $\mu(N)=\frac{1}{2} N^{3} \prod_{p \mid N, p: \text { prime }}\left(1-p^{-2}\right)$. Then $H / \Gamma(N)$ has $t=\mu(N) / N$ cusps. Let

$$
\Delta(N)=(H / \Gamma(N)) \cup\{t \text { cusps }\} .
$$

Then

$$
\begin{aligned}
& g(\Delta(N))=1+\frac{(N-6) \mu(N)}{12 N}, \\
& 2 g(\Delta(N))-2=\frac{(N-6) \mu(N)}{6 N} .
\end{aligned}
$$

Let $\Phi: B(N) \rightarrow \Delta(N)$ be the elliptic modular surface attached to $\Gamma(N)$, which is called the elliptic modular surface of level $N$. Then

$$
K_{B(N)}=\Phi^{*}(\mathfrak{f}-\mathfrak{f})
$$

where

$$
\mathfrak{i}=\text { the canonical line bundle of } \Delta(N) \text {, }
$$$$
\mathfrak{f}=\text { a line bundle on } \Delta(N) \text { with } \operatorname{deg} f=-\left(p_{g}(B(N))-q(B(N))+1\right) .
$$

We know the following:

$$
\begin{gathered}
q(B(N))=g(\Delta(N))=1+\frac{(N-6) \mu(N)}{12 N}, \\
K_{B(N)}^{2}=0,
\end{gathered}
$$




$$
\begin{gathered}
e(B(N))=\text { the Euler number of } B(N)=N t=\mu(N), \\
p_{g}(B(N))-q(B(N))+1=\frac{K_{B(N)}^{2}+e(B(N))}{12}=\frac{\mu(N)}{12}, \\
p_{g}(B(N))=\frac{(N-3) \mu(N)}{6 N}, \\
\operatorname{deg} \mathfrak{f}=-\frac{\mu(N)}{12}, \quad \operatorname{deg} \mathfrak{f}=2 g-2=\frac{(N-6) \mu(N)}{6 N}, \\
\operatorname{deg}(\mathfrak{f}-\mathfrak{f})=\frac{N-4}{4 N} \mu(N) .
\end{gathered}
$$

On the fibres of $\Phi$, we know

$$
\Phi^{-1}(v)= \begin{cases}\text { a non-singular elliptic curve } & \text { if } v \neq \text { cusp } \\ \sum_{i=0}^{N-1} \Theta_{v, i} & \text { if } v=\text { cusp }\end{cases}
$$

where $\Theta_{v, i}$ is a non-singular rational curve with $\Theta_{v, i}^{2}=-2$ and with the configuration as in Fig. 1. $B(N)$ has exactly $N^{2}$ sections

$$
\Gamma(i, j), \quad i, j=0, \cdots, N-1
$$

where $\Gamma(0,0)=$ the zero-section and as in Fig. 2

$$
\Gamma(i, j) \cdot \Theta_{\infty, k}=\left\{\begin{array}{lll}
1 & \text { if } & i=k \\
0 & \text { if } & i \neq k
\end{array}\right.
$$

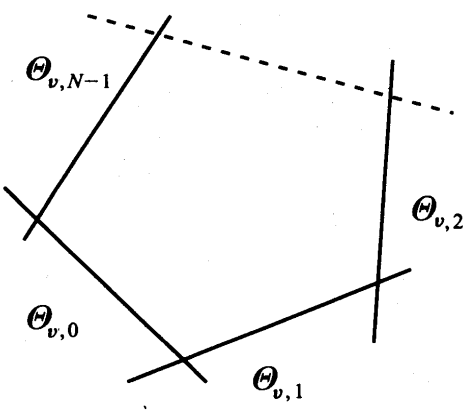

FIGURE 1

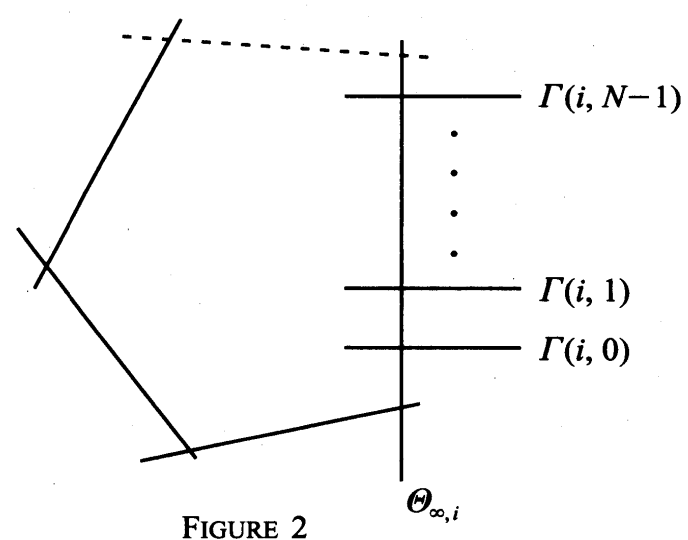

FIGURE 2

$\Gamma(i, j)$ 's are mutually disjoint and

$$
\{\Gamma(i, j) \cap F\}=\{N \text {-division points on } F\}
$$

where $F$ is a general fibre of $\Phi: B(N) \rightarrow \Delta(N)$. We know that 


$$
\begin{gathered}
K_{B(N)} \cdot \Gamma(i, j)=\operatorname{deg}(\mathfrak{f}-\mathfrak{f})=\frac{N-4}{4 N} \mu(N), \\
g(\Gamma(i, j))=g(\Delta(N))=1+\frac{(N-6) \cdot \mu(N)}{12 N}, \\
\Gamma(i, j)^{2}=\operatorname{deg} \mathfrak{f}=-\frac{\mu(N)}{12}
\end{gathered}
$$

Let

$$
\Gamma=\sum_{i, j} \Gamma(i, j) .
$$

Then $\Gamma$ is a non-singular (reducible) curve on $B(N)$,

$$
\Gamma \cap F=\{N \text {-division points on } F\} \sim N^{2}\left[0_{F}\right]
$$

where $\sim$ is the linear equivalence relation and

$$
\Gamma \cap \Theta_{v, i}=\left\{N \text {-division points on } C^{*}=P^{1}-\{0,1\}\right\}
$$

where $\Theta_{v, i}=P^{1}, \Theta_{v, i} \cap \Theta_{v, i-1}=0, \Theta_{v, i} \cap \Theta_{v, i+1}=\infty$.

LEMMA OF T. SHIODA. Let $F$ be a general fibre of $\Phi: B(N) \rightarrow \Delta(N)$ and let $D$ be a divisor on $B(N)$ such that $D \mid F \sim 0$. Then

$$
D \approx(D \cdot \Gamma(0,0)) \cdot F+\sum_{v: \text { cusp }}\left(\Theta_{v, 1}, \cdots, \Theta_{v, N-1}\right) \cdot A_{N}^{-1}\left(\begin{array}{c}
D \cdot \Theta_{v, 1} \\
\cdot \\
\cdot \\
D \cdot \Theta_{v, N-1}
\end{array}\right)
$$

where $\approx$ is the algebraic equivalence relation and

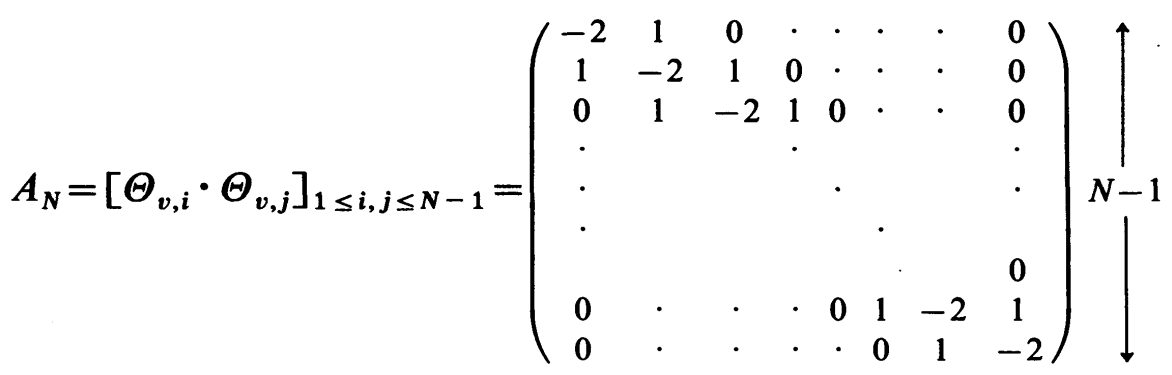

and the components of $A_{N}^{-1}\left(\begin{array}{c}D \cdot \Theta_{v, 1} \\ \dot{D} \cdot \Theta_{v, N-1}\end{array}\right)$ are integers.

Proof. Take $h$ general fibres $F_{1}, F_{2}, \cdots, F_{h}$ where $F_{i} \neq F_{j}(i \neq j)$. Then 


$$
\begin{gathered}
\rightarrow H^{1}\left(B(N), \mathcal{O}\left(D-\sum_{i=1}^{h} F_{i}\right)\right) \rightarrow H^{1}(B(N), \mathcal{O}(D)) \\
\rightarrow H^{1}\left(F_{1}, \mathcal{O}\right) \oplus H^{1}\left(F_{2}, \mathcal{O}\right) \oplus \cdots \oplus H^{1}\left(F_{h}, \mathcal{O}\right) \rightarrow H^{2}\left(B(N), \mathcal{O}\left(D-\sum_{i=1}^{h} F_{i}\right)\right) \rightarrow
\end{gathered}
$$

where $H^{1}\left(F_{i}, \mathcal{O}\right) \cong C$. Hence, if $h$ is sufficiently large,

$$
H^{2}\left(B(N), \mathcal{O}\left(D-\sum_{i=1}^{h} F_{i}\right)\right) \neq 0 \text {. }
$$

Since $H^{2}\left(B(N), \mathcal{O}\left(D-\sum_{i=1}^{h} F_{i}\right)\right) \cong H^{0}\left(B(N), \mathcal{O}\left(K+\sum_{i=1}^{h} F_{i}-D\right)\right)$, there exists an effective divisor $D^{\prime} \in\left|K+\sum_{i=1}^{h} F_{i}-D\right|$. Namely

$$
K+\sum_{i=1}^{h} F_{i}-D \sim D^{\prime}
$$

For any fibre $F$ of $\Phi: B(N) \rightarrow \Delta(N)$,

$$
D^{\prime} \cdot F=K \cdot F+\sum_{i=1}^{h} F_{i} \cdot F-D \cdot F=0
$$

Hence $D^{\prime}=\sum_{\alpha} m_{\alpha} D_{\alpha}$ where $D_{\alpha}$ 's are irreducible curves contained in the fibres of $\Phi$. Since

$$
K \approx \frac{N-4}{4 N} \mu(N) \cdot F, \quad F_{i} \approx F, \quad \sum_{i=0}^{N-1} \Theta_{v, i} \approx F
$$

we obtain

$$
D \approx p F+\sum_{i=1, v: \text { cusp }}^{N-1} q_{v, i} \Theta_{v, i}
$$

for some $p, q_{v, i} \in Z$. Since $\Gamma(0,0) \cdot \Theta_{v, i}=1$ if $i=0$ and $\Gamma(0,0) \cdot \Theta_{v, i}=0$ if $i=1, \cdots, N-1$, we obtain

$$
D \cdot \Gamma(0,0)=p F \cdot \Gamma(0,0)+\sum_{i=1}^{N-1} q_{v, i} \Theta_{v, i} \cdot \Gamma(0,0)=p .
$$

Since $F \cdot \Theta_{v, j}=0$,

$$
D \cdot \Theta_{v, j}=\sum_{i=1}^{N-1} q_{v, i} \Theta_{v, i} \cdot \Theta_{v, j}
$$

for $j=1,2, \cdots, N-1$. Let $A_{N}=\left[\Theta_{v, i} \cdot \Theta_{v, j}\right]_{1 \leq i, j \leq N-1}$. Then $A_{N}$ is non-singular and

$$
\left(\begin{array}{c}
q_{v, 1} \\
\cdot \\
\cdot \\
q_{v, N-1}
\end{array}\right)=A_{N}^{-1}\left(\begin{array}{c}
D \cdot \Theta_{v, 1} \\
\cdot \\
D \cdot \Theta_{v, N-1}
\end{array}\right)
$$


Thus

$$
D \approx(D \cdot \Gamma(0,0)) F+\sum_{v: \text { cusp }}\left(\Theta_{v, 1}, \cdots, \Theta_{v, N-1}\right) A_{N}^{-1}\left(\begin{array}{c}
D \cdot \Theta_{v, 1} \\
\cdot \\
D \cdot \Theta_{v, N-1}
\end{array}\right) .
$$

LeMMA 1-1. Let $A_{N}^{-1}=\left[x_{j k}\right]$. Then

(i) $\quad x_{j k}= \begin{cases}\frac{-j(N-k)}{N}, & j \leqq k \\ \frac{-k(N-j)}{N}, & j>k .\end{cases}$

(ii) $\quad \sum_{k=1}^{N-1} x_{j k}=\frac{-j(N-j)}{2}= \begin{cases}-m j+\frac{j(j+1)}{2}-\frac{j}{2} & \text { if } N=2 m, \\ -m j+\frac{j(j-1)}{2} & \text { if } N=2 m+1 .\end{cases}$

LEMMA $1-2 . \quad \mu(N) / 12$ is divisible by $N$ if $N \geqq 5$.

Proof. Let $\sigma(N)=N^{2} \prod_{p \mid N, p \text { : prime }}\left(1-p^{-2}\right)$. It is sufficient to prove that $24 \mid \sigma(N)$.

(i) Assume $p \geqq 5$ and $p$ is prime. Then (a) $p=3 h+1, h=2 m, m \geqq 1$, or (b) $p=3 h+2$, $h=2 m+1, m \geqq 0$.

(a) $\sigma(p)=p^{2}\left(1-p^{-2}\right)=(p+1)(p-1)=12(3 m+1) m$. Since $3 m+1$ or $m$ is even, $24 \mid \sigma(p)$.

(b) $\sigma(p)=(p+1)(p-1)=12(m+1)(3 m+2)$. Since $m+1$ or $3 m+2$ is even, $24 \mid \sigma(p)$.

(ii) Assume $N=p_{1}^{h_{1}} \cdots p_{r}^{h_{r}}, p_{i} \neq p_{j}(i \neq j), p_{i}$ :prime, $h_{i} \geqq 1$ where some $p_{i} \neq 2,3$. Then $p_{i} \geqq 5$ and, hence by (i), $24 \mid \sigma\left(p_{i}\right)$. Since

$$
\begin{gathered}
\sigma(N)=\sigma\left(p_{1}^{h_{1}}\right) \cdots \sigma\left(p_{r}^{h_{r}}\right), \\
\sigma\left(p_{i}^{h_{i}}\right)=p_{i}^{2 h_{i}}\left(1-p_{i}^{-2}\right)=p_{i}^{2 h_{i}-2} \cdot \sigma\left(p_{i}\right),
\end{gathered}
$$

we get $24 \mid \sigma(N)$.

(iii) Finally we assume $N=2^{h_{1}} \cdot 3^{h_{2}} \geqq 5$.

(a) In case $h_{1}, h_{2} \geqq 1$,

$$
\begin{aligned}
\sigma(N) & =\sigma\left(2^{h_{1}}\right) \cdot \sigma\left(3^{h_{2}}\right)=2^{2 h_{1}}\left(1-2^{-2}\right) 3^{2 h_{2}}\left(1-3^{-2}\right) \\
& =2^{2 h_{1}-2} \cdot 3^{2 h_{2}-2}\left(2^{2}-1\right)\left(3^{2}-1\right)=24 \cdot 2^{2 h_{1}-2} \cdot 3^{2 h_{2}-2} .
\end{aligned}
$$

Hence $24 \mid \sigma(N)$.

(b) In case $h_{1}=0$ and $h_{2} \geqq 2$,

$$
\sigma(N)=\sigma\left(3^{h_{2}}\right)=3^{2 h_{2}-2}\left(3^{2}-1\right)=3^{2 h_{2}-2} \cdot 8=24 \cdot 3^{2 h_{2}-3},
$$

where $2 h_{2}-3>0$. Hence $24 \mid \sigma(N)$. 
(c) In case $h_{1} \geqq 3$ and $h_{2}=0$,

$$
\sigma(N)=\sigma\left(2^{h_{1}}\right)=2^{2 h_{1}-2}\left(2^{2}-1\right)=2^{2 h_{1}-2} \cdot 3=24 \cdot 2^{2 h_{1}-5}
$$

where $2 h_{1}-5>0$. Hence $24 \mid \sigma(N)$.

Q.E.D.

REMARK 1. This is not true if $N \leqq 4$.

$$
\begin{array}{lll}
N=4: & \mu(4)=24, & \mu(4) / 12=2, \\
N=3: & \mu(3)=12, & \mu(3) / 12=1 .
\end{array}
$$

LeMMA 1-3.

$$
\Gamma \approx N^{2} \cdot \Gamma(0,0)-\left(1-N^{2}\right) \frac{\mu(N)}{12} F-\sum_{v: \text { cusp, } j=1, \cdots, N-1} \frac{j(N-j)}{2} N \cdot \Theta_{v, j}
$$

Proof. Let $D=\Gamma-N^{2} \cdot \Gamma(0,0)$. Then $D \mid F=0$. By the Lemma of Shioda

$$
D \approx(D \cdot \Gamma(0,0)) F+\sum_{v: \text { cusp }}\left(\Theta_{v, 1}, \cdots, \Theta_{v, N-1}\right) A_{N}^{-1}\left(\begin{array}{c}
D \cdot \Theta_{v, 1} \\
\vdots \\
D \cdot \Theta_{v, N-1}
\end{array}\right)
$$

where

$$
\begin{gathered}
D \cdot \Gamma(0,0)=\left(1-N^{2}\right) \cdot \Gamma(0,0)^{2}=-\left(1-N^{2}\right) \cdot \mu(N) / 12, \\
D \cdot \Theta_{v, i}=\Gamma \cdot \Theta_{v, i}=N \quad \text { for } \quad i=1, \cdots, N-1
\end{gathered}
$$

Thus Lemma 1-1 implies Lemma 1-3.

Q.E.D.

Lemmas 1-1, 1-2, 1-3 and the above Remark 1 imply

Proposition 1-1. Assume $N \geqq 4$. Then

$$
\Gamma=\sum_{i, j=0}^{N-1} \Gamma(i, j) \text { is divisible } \begin{cases}\text { by } N & \text { if } N \text { is odd }, \\ \text { by } N / 2 & \text { if } N \text { is even } .\end{cases}
$$

REMARK 2. In case $N=3$,

$$
\begin{gathered}
\mu(3)=12, \\
\Gamma(i, j)^{2}=-\mu(3) / 12=-1, \\
g(\Gamma(i, j))=1+(3-6) \cdot \mu(3) /(12 \cdot 3)=0 .
\end{gathered}
$$

Hence $\Gamma$ is not divisible by 3 and $\Gamma(i, j)$ 's are exceptional curves of the first kind.

REMARK 3. If $N$ is even, $\Gamma$ is not divisible by $N$. We refer to our previous paper $\left[\mathrm{I}_{1}\right]$ for a proof.

REMARK 4. In $\S 1$ of [S], Shioda remarked that the Néron-Severi group $N S(B(N))$ 
is torsion-free. This fact can be proved as follows:

Proof of Remark 4. Let $D$ be a divisor on $B(N)$ such that $D \neq 0$ and $n D \approx 0$ for some positive integer $n$. Then

$$
\sum_{\nu=0}^{2}(-1)^{v} \operatorname{dim} H^{v}(B(N), \mathcal{O}(D))=p_{g}-q+1=\frac{\mu(N)}{12} \geqq 1
$$

where $H^{0}(B(N), \mathcal{O}(D))=0$ and $H^{2}(B(N), \mathcal{O}(D)) \cong H^{0}\left(B(N), \mathcal{O}\left(K_{B(N)}-D\right)\right)$. Hence there exists an effective divisor $D^{\prime} \in\left|K_{B(N)}-D\right|$. Since $D^{\prime}$ is effective and $D^{\prime} \cdot F=K_{B(N)} \cdot F-$ $D \cdot F=0$, we obtain that $D^{\prime} \mid F \sim 0$. By the Lemma of Shioda and by the fact that $D^{\prime} \cdot \Theta_{v, i}=0$, we get

$$
D^{\prime} \approx\left(D^{\prime} \cdot \Gamma(0,0)\right) F \text {. }
$$

Since $K_{B(N)}=\Phi^{*}(\mathfrak{f}-f)$, we obtain that $D \approx h F$ for some integer $h$. Since $n D \approx 0$, $0=D \cdot \Gamma(0,0)=h \cdot F \cdot \Gamma(0,0)=h$. Thus $h=0$ and $D \approx 0 \cdot F=0$.

\section{§2. The example $A(N, n)$.}

From now on, we assume $N \geqq 4$. By Proposition $1-1, \Gamma=\sum_{i, j=0}^{N-1} \Gamma(i, j)$ is divisible by $N$ if $N$ is odd, and by $N / 2$ if $N$ is even. Let $n$ be an integer such that $n \geqq 2$ and

$$
\begin{array}{ll}
n \mid N & \text { if } N \text { is odd, } \\
n \mid(N / 2) & \text { if } N \text { is even. }
\end{array}
$$

Then $[\Gamma]=n L$ for some line bundle $L \in H^{1}\left(B(N), \mathcal{O}^{*}\right)$. Hence we can construct, in the bundle space of $L$, an $n$-fold branched covering

$$
\varphi: A(N, n) \rightarrow B(N)
$$

along a non-singular branch locus $\Gamma(\subset B(N))$.

Let

$K_{S}=$ the canonical line bundle of a compact complex surface $S$ (the canonical divisor of $S$ is also denoted by $K_{s}$ ),

$e(X)=$ the topological Euler number of a space $X$,

$\tau(S)=$ the topological index of $S=\left(K_{S}^{2}-2 e(S)\right) / 3$,

$p_{g}(S)=$ the geometric genus of $S, q(S)=$ the irregularity of $S$.

We have the following classically known

LEMMA 2-1. Let $\varphi: A \rightarrow B$ be an $n$-fold branched covering along a non-singular branch locus $\Gamma(\subset B)$. Then 
(i) $\quad K_{A}=\varphi^{*} K_{B}+(n-1) \Gamma^{*}$

where $n \Gamma^{*}=\varphi^{*} \Gamma$ and $\Gamma^{*}=\varphi^{-1}(\Gamma)$. Hence

$$
K_{A}^{2}=n K_{B}^{2}+2(n-1) K_{B} \cdot \Gamma+\frac{(n-1)^{2}}{n} \Gamma^{2} .
$$

(ii) $\quad e(A)=n e(B)-(n-1) e(\Gamma)$.

(iii) $\quad p_{g}(S)-q(S)+1=\frac{K_{S}^{2}+e(S)}{12} \quad$ (Noether's formula)

In the following, we shall calculate some numerical invariants of $A(N, n)$.

Proposition 2-1.

$$
\begin{gathered}
K_{A(N, n)}^{2}=\frac{N(n-1)\{(5 n+1) N-24 n\}}{12 n} \cdot \mu(N), \\
e(A(N, n))=\frac{6 n+(n-1) N(N-6)}{6} \cdot \mu(N) .
\end{gathered}
$$

Proof. By Lemma 2-1,

$$
K_{A(N, n)}^{2}=n K_{B(N)}^{2}+2(n-1) K_{B(N)} \cdot \Gamma+\frac{(n-1)^{2}}{n} \Gamma^{2}
$$

where

$$
\begin{gathered}
K_{B(N)}^{2}=0, \\
K_{B(N)} \cdot \Gamma=\sum_{i, j} K_{B(N)} \cdot \Gamma(i, j)=N^{2} \cdot \frac{N-4}{4 N} \mu(N)=\frac{N(N-4)}{4} \mu(N), \\
\Gamma^{2}=\sum_{i, j} \Gamma(i, j)^{2}=N^{2} \frac{-\mu(N)}{12}=-\frac{N^{2}}{12} \mu(N) .
\end{gathered}
$$

Hence

$$
\begin{aligned}
K_{A(N, n)}^{2} & =2(n-1) \cdot \frac{N(N-4)}{4} \cdot \mu(N)-\frac{(n-1)^{2}}{n} \frac{N^{2}}{12} \mu(N) \\
& =\frac{N(n-1)\{(5 n+1) N-24 n\}}{12 n} \cdot \mu(N) .
\end{aligned}
$$

By Lemma 2-1,

$$
e(A(N, n))=n e(B(N))-(n-1) e(\Gamma)
$$

where 


$$
\begin{gathered}
e(B(N))=\mu(N), \\
e(\Gamma)=\sum_{i, j} e(\Gamma(i, j))=N^{2} \cdot e(\Delta(N))=N^{2}(2-2 g(\Delta(N))) \\
=N^{2} \cdot 2\left(-\frac{N-6}{12 N} \cdot \mu(N)\right)=-\frac{N(N-6)}{6} \cdot \mu(N) .
\end{gathered}
$$

Hence

$$
\begin{aligned}
e(A(N, n)) & =n \cdot \mu(N)+(n-1) \frac{N(N-6)}{6} \cdot \mu(N) \\
& =\frac{6 n+(n-1) N(N-6)}{6} \mu(N) .
\end{aligned}
$$

Proposition 2-2. Assume $N \geqq 5$. Then

(i ) $\quad 3 e(A(N, n)) \geqq K_{A(N, n)}^{2} \geqq 2 e(A(N, n))$,

(ii) $\quad 3 e(A(N, n))=K_{A(N, n)}^{2}$ if and only if $(N, n)=(7,7),(8,4),(9,3),(12,2)$,

(iii) $\quad K_{A(N, n)}^{2}=2 e(A(N, n))$ if and only if $(N, n)=(5,5)$.

Proof. By Proposition 2-1

$$
3 e(A(N, n))-K_{A(N, n)}^{2}=\frac{\mu(N)}{12 n}\{(n-1) N-6 n\}^{2} \geqq 0 .
$$

The equality holds if and only if $N=6 n /(n-1)$. Since $n \geqq 2$, this is equivalent to $(N, n)=(7,7),(8,4),(9,3),(12,2)$. By Proposition 2-1

$$
K_{A(N, n)}^{2}-2 e(A(N, n))=\frac{\mu(N)}{12 n}\left\{\left(n^{2}-1\right) N^{2}-24 n^{2}\right\} .
$$

If $N=5$ (and hence $n=5)$, then

$$
K_{A(N, n)}^{2}-2 e(A(N, n))=\frac{\mu(5)}{12 \cdot 5}\left\{\left(5^{2}-1\right) \cdot 5^{2}-24 \cdot 5^{2}\right\}=0 .
$$

If $N \geqq 6$, then, since $n \geqq 2$,

$$
\begin{gathered}
K_{A(N, n)}^{2}-2 e(A(N, n)) \geqq \frac{\mu(N)}{12 n}\left\{\left(n^{2}-1\right) 36-24 n^{2}\right\} \\
=\frac{\mu(N)}{12 n}\left(12 n^{2}-36\right)=\frac{\mu(N)}{n}\left(n^{2}-3\right)>0
\end{gathered}
$$

Proposition 2-3. (i) If $N \geqq 6$, then $A(N, n)$ is a minimal surface of general type 
with positive topological index.

(ii) $A(5,5)$ is a surface of general type with $K_{A(5,5)}^{2}=200$ and $e(A(5,5))=100$. Let

$$
\Gamma^{*}(i, j)=\varphi^{-1}(\Gamma(i, j)) \quad\left(\text { hence } 5 \Gamma^{*}(i, j)=\varphi^{*} \Gamma(i, j)\right) \text {. }
$$

Then $\Gamma^{*}(i, j)^{\prime}$ 's are exceptional curves of the first kind. Let $A_{0}$ be the surface obtained by blowing down $\Gamma^{*}(i, j)$ 's. Then $A_{0}$ is a minimal surface of general type with $K_{A_{0}}^{2}=225$ and $e\left(A_{0}\right)=75\left(\right.$ and hence $\left.K_{A_{0}}^{2}=3 e\left(A_{0}\right)\right)$.

Proof. By Lemma 2-1

$$
\begin{gathered}
K_{A(N, n)}=\varphi^{*} K_{B(N)}+(n-1) \Gamma^{*}=\varphi^{*} \Phi^{*}(\mathfrak{f}-\mathfrak{f})+(n-1) \sum_{i, j} \Gamma^{*}(i, j), \\
\operatorname{dim}|\mathfrak{f}|-\operatorname{dim}|\mathfrak{f}-\mathfrak{f}|=\operatorname{deg} \mathfrak{f}+1-g(\Delta(N))=\frac{3-N}{6 N} \mu(N) .
\end{gathered}
$$

Since $\operatorname{deg} \mathfrak{f}=-\mu(N) / 12<0, \operatorname{dim}|\mathfrak{f}|=-1$. Hence

$$
\operatorname{dim}|\mathfrak{f}-\mathfrak{f}|=\frac{N-3}{6 N} \mu(N)-1>0 \quad \text { if } \quad N \geqq 5 .
$$

In particular, $p_{g}(A(N, n))>0$. If there exists an exceptional curve of the first kind on $A(N, n)$, then it is contained in the divisor $K_{A(N, n)}$ and, hence, is one of $\Gamma^{*}(i, j)$ 's, while

$$
\begin{gathered}
g\left(\Gamma^{*}(i, j)\right)=g(\Gamma(i, j))=1+\frac{(N-6) \mu(N)}{12 N}, \\
\Gamma^{*}(i, j)^{2}=\frac{\Gamma(i, j)^{2}}{n}=-\frac{\mu(N)}{12 n} .
\end{gathered}
$$

If $N \geqq 6$, then $g\left(\Gamma^{*}(i, j)\right) \geqq 1$ and hence $A(N, n)$ is minimal. If $N=5$, then $\mu(5)=$ $\frac{1}{2} 5^{3} \cdot\left(1-5^{-2}\right)=60$. Hence $g\left(\Gamma^{*}(i, j)\right)=0$ and $\Gamma^{*}(i, j)^{2}=-1$, namely, $\Gamma^{*}(i, j)$ 's are exceptional curves of the first kind. Since $\mu(5)=60, g(\Delta(5))=1+(5-6) \mu(5) /(12 \cdot 5)=0$ and $\operatorname{deg}(\mathfrak{f}-\mathfrak{f})=((5-4) /(4 \cdot 5)) \mu(5)=(1 / 20) 60=3$,

$$
K_{A(5,5)}=3 \varphi^{*} F+4 \sum_{i, j=0}^{4} \Gamma^{*}(i, j)
$$

where $F$ is a general fibre of $\Phi: B(5) \rightarrow \Delta(5)$ and $F \cdot \Gamma^{*}(i, j)=1$. Hence $K_{A_{0}}=3 F_{*}$ where $F_{*}$ is a non-singular curve with $g\left(F_{*}\right)=11$. In particular $A_{0}$ is minimal. Since $K_{A(5,5)}^{2}=200$ and $e(A(5,5))=100$ by Proposition $2-1, A_{0}$ is a minimal surface of general type with $K_{A_{0}}^{2}=225$ and $e\left(A_{0}\right)=75$. By Proposition 2-1,

$$
K_{A(N, n)}^{2}=\frac{N(n-1)\{(5 n+1) N-24 n\}}{12 n} \cdot \mu(N)
$$




$$
\begin{aligned}
& \geqq \frac{N(n-1)\{(5 n+1) 5-24 n\}}{12 n} \cdot \mu(N) \\
& =\frac{N(n-1)(n+5)}{12 n} \cdot \mu(N)>0
\end{aligned}
$$

for $N \geqq 5$. Thus $A(N, n)$ is of general type. The topological index $\tau(A(N, n))=$ $\left(K_{A(N, n)}^{2}-2 e(A(N, n))\right) / 3$ is positive if $N \geqq 6$ by Proposition $2-2$.

Q.E.D. know

REMARK 1. On the geometric genus $p_{g}\left(A_{0}\right)$ and the irregularity $q\left(A_{0}\right)$ of $A_{0}$, we

$$
p_{g}\left(A_{0}\right)=34, \quad q\left(A_{0}\right)=10 .
$$

As for the detailed calculations, we refer to $\left[\mathrm{I}_{1}\right]$.

REMARK 2. In case $N=4$ and $n=2, B(4)$ is a $K 3$ surface and $\Gamma(i, j)^{2}=-2$, $g(\Gamma(i, j))=0$. Let $\Gamma^{*}(i, j)=\varphi^{-1}(\Gamma(i, j))$. Then

$$
\begin{gathered}
K_{A(4,2)}=\sum_{i, j} \Gamma^{*}(i, j), \\
\Gamma^{*}(i, j)^{2}=-1, \quad g\left(\Gamma^{*}(i, j)\right)=0, \\
K_{A(4,2)}^{2}=-16, \quad e(A(4,2))=16
\end{gathered}
$$

by Lemma $2-1$ and Proposition 2-1. Let $A_{0}(4,2)$ be the surface obtained by blowing down $\Gamma^{*}(i, j)$ 's. Then

$$
K_{A_{0}(4,2)}=0, \quad e\left(A_{0}(4,2)\right)=0 .
$$

This implies that $A_{0}(4,2)$ is an abelian surface and $B(4)$ is a Kummer surface.

REMARK 3. Fix $n \geqq 2$ and consider $N$ 's which are multiples of $n$. Then, by Proposition 2-1,

$$
\lim _{N \rightarrow \infty} \frac{K_{A(N, n)}^{2}}{e(A(N, n))}=\frac{5 n+1}{2 n} .
$$

Moreover $K_{A(N, n)}^{2} / e(A(N, n))>5 / 2$ if and only if $(N, n) \neq(5,5)$.

REMARK 4. (i) The canonical line bundle of $A(N, n), N \geqq 6$, and of $A_{0}$ are ample.

(ii) $A(6,3)$ contains elliptic curves $\Gamma^{*}(i, j)$. Hence its universal covering space is not a bounded domain, while

$$
\frac{K_{A(6,3)}^{2}}{e(A(6,3))}=\frac{8}{3}=2.66 \cdots
$$

REMARK 5. There exist some other congruence relations between $\Gamma(i, j)$ 's and 
$\Theta_{v, i}$ 's. For instance, in the case $N=2 m$ (even), $\Gamma_{e}=\sum_{i, j: \text { even }} \Gamma(i, j)$ is divisible by $m$ if $m$ is odd and divisible by $m / 2$ if $m$ is even. Hence we can construct other branched coverings of $B(N)$ corresponding to them. We refer to $\left[\mathrm{I}_{1}\right]$ for details.

REMARK 6. E. Horikawa also gave another series of surfaces of general type with positive indices as branched coverings of the product of two algebraic curves. His construction is very simple but has a close relation with the moduli of algebraic curves. We refer also to $\left[I_{1}\right]$ for details.

\section{PART II Surfaces of General Type with $p_{g}=0$}

\section{§3. Hypersurfaces of the product of three elliptic curves.}

We denote by $\theta_{1}$ and $\theta_{2}$ the usual theta functions, namely,

$$
\begin{aligned}
& \theta_{1}(z)=2\left(\sum_{n=1}^{\infty}(-1)^{n-1} q^{((2 n-1) / 2)^{2}} \sin (2 n-1) \pi z\right) \\
& \theta_{2}(z)=2\left(\sum_{n=1}^{\infty} q^{((2 n-1) / 2)^{2}} \cos (2 n-1) \pi z\right)
\end{aligned}
$$

where $\tau \in C, \operatorname{Im} \tau>0$ and $q=\exp (\pi \sqrt{-1} z)$. Then as is classically known we have

LEMMA 3-1.

$$
\begin{array}{ll}
\theta_{1}(z+1)=-\theta_{1}(z), & \theta_{2}(z+1)=-\theta_{2}(z), \\
\theta_{1}(z+\tau)=-\delta \theta_{1}(z), & \theta_{2}(z+\tau)=\delta \theta_{2}(z), \\
\theta_{1}\left(z+\frac{1}{2}\right)=\theta_{2}(z), & \theta_{2}\left(z+\frac{1}{2}\right)=-\theta_{1}(z), \\
\theta_{1}(-z)=-\theta_{1}(z), & \theta_{2}(-z)=\theta_{2}(z),
\end{array}
$$

where $\delta=\exp (\pi \sqrt{-1}(2 z+\tau))$.

In particular, $\left(\theta_{1}\right)^{2}$ and $\left(\theta_{2}\right)^{2}$ are sections of a line bundle $2[o]$ on the elliptic curve $E=C /\langle 1, \tau\rangle$ with periods $1, \tau$ where $o$ is the origin of $E$.

Let $\wp(z)$ be the $\wp$-function, namely,

$$
\wp(z)=\wp(z, \tau)=\frac{\theta_{2}(z)^{2}-\theta_{1}(z)^{2}}{\theta_{2}(z)^{2}+\theta_{1}(z)^{2}} .
$$

Then we have the following also well-known

LEMMA 3-2.

$$
\begin{gathered}
\wp(z)=\wp(z+1)=\wp(z+\tau), \quad \wp\left(z+\frac{1}{2}\right)=-\wp(z), \\
\wp(-z)=\wp(z), \quad \wp(z+\tau / 2)=a / \wp(z),
\end{gathered}
$$

where $a=\wp(\tau / 2)$ can take any value $\in C-\{0, \pm 1\}$. In particular, $\wp(z)$ is a meromorphic 
function on the elliptic curve $E$.

(ii) $\wp\left(\frac{1}{2}\right)=-1, \quad \wp(0)=1, \quad \wp(\tau / 2)=a, \quad \wp((1+\tau) / 2)=-a$.

(iii) $\quad \frac{d \wp}{d z}(z)=0 \quad$ if and only if $z=0, \frac{1}{2}, \tau / 2,(1+\tau) / 2$.

In particular, $\wp: E \rightarrow \boldsymbol{P}^{1}$ is a double covering ramified over $\pm 1, \pm a \in \boldsymbol{P}^{1}$.

(iv) Let $b=\wp(\tau / 4)$. Then $b^{2}=a$.

Now we take three elliptic curves $E_{i}=C /\left\langle 1, \tau_{i}\right\rangle, i=1,2,3$. Let $\left(z_{1}, z_{2}, z_{3}\right)$ be the coordinates on the product $E_{1} \times E_{2} \times E_{3}$ and

$$
\begin{gathered}
o_{i}=\text { the origin of } E_{i}, \quad \wp_{i}\left(z_{i}\right)=\wp\left(z_{i}, \tau_{i}\right), \\
a_{i}=\wp_{i}\left(\tau_{i} / 2\right), \quad b_{i}=\wp_{i}\left(\tau_{i} / 4\right) \quad\left(b_{i}^{2}=a_{i}\right)
\end{gathered}
$$

for $i=1,2,3$. For any $c \in C^{*}$, we define the subvariety $X_{c}$ by

$$
X_{c}=\left\{\left(z_{1}, z_{2}, z_{3}\right) \in E_{1} \times E_{2} \times E_{3} \mid \wp_{1}\left(z_{1}\right) \cdot \wp_{2}\left(z_{2}\right) \cdot \wp_{3}\left(z_{3}\right)=c\right\} .
$$

Let $\psi_{i}: E_{1} \times E_{2} \times E_{3} \rightarrow E_{i}$ be the projection to the $i$-th factor. Then

$$
\left[X_{c}\right]=\psi_{1}^{*} 2\left[o_{1}\right] \otimes \psi_{2}^{*} 2\left[o_{2}\right] \otimes \psi_{3}^{*} 2\left[o_{3}\right]
$$

and $\left[X_{c}\right]$ is ample on $E_{1} \times E_{2} \times E_{3}$. By the theorem of Bertini, $X_{c}$ is irreducible. By Lemma 3-2, the singular points of $X_{c}$ are isolated and at most ordinary double points,

\{singular points of $\left.X_{c}\right\}=X_{c} \cap\left\{2\right.$-division points on $\left.E_{1} \times E_{2} \times E_{3}\right\}$, and moreover, if $\left(z_{1}, z_{2}, z_{3}\right)$ is a singular point on $X_{c}$, then

$$
\left(z_{1}+\frac{1}{2}, z_{2}+\frac{1}{2}, z_{3}\right), \quad\left(z_{1}, z_{2}+\frac{1}{2}, z_{3}+\frac{1}{2}\right), \quad\left(z_{1}+\frac{1}{2}, z_{2}, z_{3}+\frac{1}{2}\right)
$$

are also singular points on $X_{c}$. Let

$$
\mathscr{E}=\left\{\text { the values of } \wp_{1}\left(z_{1}\right) \cdot \wp_{2}\left(z_{2}\right) \cdot \wp_{3}\left(z_{3}\right) \text { on the } 2 \text {-division points }\right\}
$$

Then

$$
\mathscr{E}=\left\{ \pm 1, \pm a_{i}, \pm a_{i} \cdot a_{j}, \pm a_{1} \cdot a_{2} \cdot a_{3},(i \neq j, i, j=1,2,3)\right\} .
$$

Let $n_{c}$ be the number of the singular points on $X_{c}$. Then by elementary calculations we obtain the following:

(0) The possible values of $n_{c}$ are $0,4,8,12$ and 16. $n_{c}$ really takes these values. For instance

(1) If $c \notin \mathscr{E}$, then $n_{c}=0$, namely, $X_{c}$ is non-singular.

(2) If $c \in \mathscr{E}$ and $E_{i}$ 's are general, then $n_{c}=4$.

(3) If $c \in \mathscr{E}$ and

$$
c= \pm a_{i}, \quad a_{i}= \pm a_{j} \cdot a_{k}, \quad((i, j, k) \text { is a permutation of }(1,2,3))
$$


or

$$
c= \pm 1, \quad a_{i} \cdot a_{j}= \pm 1 \quad \text { for some } i, j(i \neq j)
$$

then $n_{c}=8$.

(4) If $c \in \mathscr{E}$ and

$$
c=a_{i} \text { for some } i, \quad a_{j}= \pm a_{i}(\neq \pm \sqrt{-1}) \quad(\text { for any } j \neq i),
$$

then $n_{c}=12$.

(5) If $c \in \mathscr{E}$ and

$$
c= \pm 1 \quad \text { or } \quad \pm \sqrt{-1}, \quad a_{i}= \pm \sqrt{-1} \quad(i=1,2,3),
$$

then $n_{c}=16$.

Thus we obtain

Proposition 3-1. The subvariety $X_{c}$ is irreducible and non-singular outside exactly $n_{c}$ ordinary double points where

$$
n_{c}=0,4,8,12 \text { or } 16 \text {. }
$$

Let $\imath: \tilde{X}_{c} \rightarrow X_{c} \subset E_{1} \times E_{2} \times E_{3}$ be the minimal resolution of $X_{c}$. We shall calculate some numerical invariants of $\tilde{X}_{c}$ in the following

Proposition 3-2. (i) $\tilde{X}_{c}$ is a minimal surface of general type with the numerical invariants:

$$
p_{g}\left(\tilde{X}_{c}\right)=10, \quad q\left(\tilde{X}_{c}\right)=3, \quad K_{\tilde{X}_{c}}^{2}=e\left(\tilde{X}_{c}\right)=48 .
$$

(ii) $l$ induces an isomorphism between the spaces of holomorphic 1-forms

$$
\imath^{*}: \dot{H}^{\mathrm{o}}\left(E_{1} \times E_{2} \times E_{3}, \Omega^{1}\right) \rightarrow H^{0}\left(\tilde{X}_{c}, \Omega^{1}\right) \text {. }
$$

Proof. (i) Minimality is clear from the construction. Since $\tilde{X}_{c}$ 's are homeomorphic to each other and the numerical invariants $p_{g}, q, K^{2}$ and $e$ are homologically invariant, we may assume that $\tilde{X}_{c}=X_{c}$, namely, $X_{c}$ is non-singular. By the adjunction formula,

$$
K_{X_{c}}=\left(K_{E_{1} \times E_{2} \times E_{3}}+\left[X_{c}\right]\right)\left|X_{c}=\left[X_{c}\right]\right| X_{c}
$$

Since

$$
\begin{aligned}
{\left[X_{c}\right] } & =\psi_{1}^{*} 2\left[o_{1}\right] \otimes \psi_{2}^{*} 2\left[o_{2}\right] \otimes \psi_{3}^{*} 2\left[o_{3}\right] \\
& =2\left[o_{1} \times E_{2} \times E_{3}+E_{1} \times o_{2} \times E_{3}+E_{1} \times E_{2} \times o_{3}\right]
\end{aligned}
$$

and

$$
K_{X_{c}}^{2}=\left\{\begin{array}{lll}
{\left[X_{c}\right]^{2}} & \text { on } X_{c} \\
{\left[X_{c}\right]^{3}} & \text { on } & E_{1} \times E_{2} \times E_{3},
\end{array}\right.
$$


we obtain

$$
K_{X_{c}}^{2}=8 \times 6=48 .
$$

From the short exact sequence

$$
0 \rightarrow \mathcal{O}_{E_{1} \times E_{2} \times E_{3}} \rightarrow \mathcal{O}_{E_{1} \times E_{2} \times E_{3}}\left(\left[X_{c}\right]\right) \rightarrow \mathcal{O}_{X_{c}}\left(K_{X_{c}}\right) \rightarrow 0,
$$

it follows

$$
\begin{aligned}
0 & \rightarrow H^{0}\left(E_{1} \times E_{2} \times E_{3}, \mathcal{O}_{E_{1} \times E_{2} \times E_{3}}\right) \rightarrow H^{0}\left(E_{1} \times E_{2} \times E_{3}, \mathcal{O}_{E_{1} \times E_{2} \times E_{3}}\left(\left[X_{c}\right]\right)\right) \\
& \rightarrow H^{0}\left(X_{c}, \mathcal{O}_{X_{c}}\left(K_{X_{c}}\right)\right) \rightarrow H^{1}\left(E_{1} \times E_{2} \times E_{3}, \mathcal{O}_{E_{1} \times E_{2} \times E_{3}}\right) \\
& \rightarrow H^{1}\left(E_{1} \times E_{2} \times E_{3}, \mathcal{O}_{E_{1} \times E_{2} \times E_{3}}\left(\left[X_{c}\right]\right)\right) \rightarrow H^{1}\left(X_{c}, \mathcal{O}_{X_{c}}\left(K_{X_{c}}\right)\right) \\
& \rightarrow H^{2}\left(E_{1} \times E_{2} \times E_{3}, \mathcal{O}_{E_{1} \times E_{2} \times E_{3}}\right) \rightarrow H^{2}\left(E_{1} \times E_{2} \times E_{3}, \mathcal{O}_{E_{1} \times E_{2} \times E_{3}}\left(\left[X_{c}\right]\right)\right) \rightarrow .
\end{aligned}
$$

Since $\left[X_{c}\right]$ is ample on $E_{1} \times E_{2} \times E_{3}$,

$$
\operatorname{dim} H^{i}\left(E_{1} \times E_{2} \times E_{3}, \mathcal{O}_{E_{1} \times E_{2} \times E_{3}}\left(\left[X_{c}\right]\right)\right)=0 \quad \text { for } i \geqq 1 .
$$

From the formula of Künneth, it follows

$$
\begin{aligned}
& \operatorname{dim} H^{v}\left(E_{1} \times E_{2} \times E_{3}, \mathcal{O}_{E_{1} \times E_{2} \times E_{3}}\right) \\
&= \sum_{i+j+k=v} \operatorname{dim} H^{i}\left(E_{1}, \mathcal{O}_{E_{1}}\right) \cdot \operatorname{dim} H^{j}\left(E_{2}, \mathcal{O}_{E_{2}}\right) \cdot \operatorname{dim} H^{k}\left(E_{3}, \mathcal{O}_{E_{3}}\right) \\
& \quad= \begin{cases}1 & \text { for } \quad v=0,3 \\
3 & \text { for } \quad v=1,2 \\
0 & \text { for } \quad v>3,\end{cases} \\
& \operatorname{dim} H^{0}\left(E_{1} \times E_{2} \times E_{3}, \mathcal{O}_{E_{1} \times E_{2} \times E_{3}}\left(\left[X_{c}\right]\right)\right) \\
&=\operatorname{dim} H^{0}\left(E_{1} \times E_{2} \times E_{3}, \mathcal{O}_{E_{1} \times E_{2} \times E_{3}}\left(\psi_{1}^{*} 2\left[o_{1}\right] \otimes \psi_{2}^{*} 2\left[o_{2}\right] \otimes \psi_{3}^{*} 2\left[o_{3}\right]\right)\right. \\
&=\prod_{i=1}^{3} \operatorname{dim} H^{0}\left(E_{i}, \mathcal{O}_{E_{i}}\left(\psi_{i}^{*} 2\left[o_{i}\right]\right)\right)=2 \cdot 2 \cdot 2=8 .
\end{aligned}
$$

Thus we obtain

$$
\begin{aligned}
p_{g}\left(X_{c}\right)= & \operatorname{dim} H^{0}\left(X_{c}, \mathcal{O}\left[K_{X_{c}}\right]\right) \\
= & \operatorname{dim} H^{1}\left(E_{1} \times E_{2} \times E_{3}, \mathcal{O}_{E_{1} \times E_{2} \times E_{3}}\right) \\
& +\operatorname{dim} H^{0}\left(E_{1} \times E_{2} \times E_{3}, \mathcal{O}_{E_{1} \times E_{2} \times E_{3}}\left(\left[X_{c}\right]\right)\right) \\
& -\operatorname{dim} H^{0}\left(E_{1} \times E_{2} \times E_{3}, \mathcal{O}_{E_{1} \times E_{2} \times E_{3}}\right) \\
= & 3+8-1=10, \\
q\left(X_{c}\right)= & \operatorname{dim} H^{1}\left(X_{c}, \mathcal{O}_{X_{c}}\right)=\operatorname{dim} H^{1}\left(X_{c}, \mathcal{O}\left[K_{X_{c}}\right]\right) \\
= & \operatorname{dim} H^{2}\left(E_{1} \times E_{2} \times E_{3}, \mathcal{O}_{E_{1} \times E_{2} \times E_{3}}\right)=3
\end{aligned}
$$


By Noether's formula (Lemma 2-1)

$$
48+e\left(X_{c}\right)=K_{X_{c}}^{2}+e\left(X_{c}\right)=12\left(p_{g}\left(X_{c}\right)-q\left(X_{c}\right)+1\right)=12(10-3+1)=96 .
$$

Hence $e\left(X_{c}\right)=48$. Since $p_{g}\left(X_{c}\right)>0$ and $K_{X_{c}}^{2}>0, X_{c}$ is a surface of general type.

(ii) Since $X_{c}$ is not linear, $\imath^{*} d z_{1}, \imath^{*} d z_{2}$ and $\imath^{*} d z_{3}$ are linearly independent. Since $d z_{1}, d z_{2}$ and $d z_{3}$ form a system of basis of $H^{0}\left(E_{1} \times E_{2} \times E_{3}, \Omega^{1}\right), \imath^{*}$ is injective, while

$$
\begin{gathered}
\operatorname{dim} H^{0}\left(E_{1} \times E_{2} \times E_{3}, \Omega^{1}\right)=3, \\
\operatorname{dim} H^{0}\left(\tilde{X}_{c}, \Omega^{1}\right)=\operatorname{dim} H^{1}\left(\tilde{X}_{c}, \mathcal{O}_{\tilde{X}_{c}}\right)=q\left(\tilde{X}_{c}\right)=3 .
\end{gathered}
$$

Hence $\imath^{*}$ is an isomorphism.

Q.E.D.

\section{§4. The example $Y_{c}$.}

We consider the following automorphisms of $E_{1} \times E_{2} \times E_{3}$ :

$$
\begin{aligned}
& g_{1}:\left(z_{1}, z_{2}, z_{3}\right) \rightarrow\left(-z_{1}+\frac{1}{2}, z_{2}+\frac{1}{2}, z_{3}\right), \\
& g_{2}:\left(z_{1}, z_{2}, z_{3}\right) \rightarrow\left(z_{1},-z_{2}+\frac{1}{2}, z_{3}+\frac{1}{2}\right), \\
& g_{3}:\left(z_{1}, z_{2}, z_{3}\right) \rightarrow\left(z_{1}+\frac{1}{2}, z_{2},-z_{3}+\frac{1}{2}\right) .
\end{aligned}
$$

Let $G$ be the group generated by $g_{1}, g_{2}$ and $g_{3}$ :

$$
G=\left\langle g_{1}, g_{2}, g_{3}\right\rangle(\cong(Z / 2 Z) \oplus(Z / 2 Z) \oplus(Z / 2 Z)) \text {. }
$$

Then, by Lemma 3-2, $X_{c}$ is invariant under the action of $G$. Thus $G$ operates on $X_{c}$ and, hence, naturally on the minimal resolution $\tilde{X}_{c}$ of $X_{c}$. Let $Y_{c}$ be the quotient surface of $\tilde{X}_{c}$ by $G: Y_{c}=\tilde{X}_{c} / G$.

PROPOSITION 4-1. $\quad Y_{c}$ is a non-singular minimal surface of general type with

$$
p_{g}\left(Y_{c}\right)=q\left(Y_{c}\right)=0, \quad K_{Y_{c}}^{2}=6-n_{c} / 4, \quad e\left(Y_{c}\right)=6+n_{c} / 4
$$

where $n_{c}=$ the number of singular points of $X_{c}=0,4,8,12$ or 16. Hence

$$
K_{Y_{c}}^{2}=6,5,4,3 \text { or } 2, \quad e\left(Y_{c}\right)=6,7,8,9 \text { or } 10
$$

according as $n_{c}=0,4,8,12$ or 16 .

OUTLINe OF THE Proof. (For details on the proof, we refer to our forthcoming paper $\left[\mathrm{I}_{2}\right]$.) Let

$$
\begin{gathered}
g_{0}=g_{1} \circ g_{2} \circ g_{3}:\left(z_{1}, z_{2}, z_{3}\right) \rightarrow\left(-z_{1},-z_{2},-z_{3}\right) \\
\bar{G}=G /\left\langle g_{0}\right\rangle, \quad \bar{Y}_{c}=\tilde{X}_{c} /\left\langle g_{0}\right\rangle .
\end{gathered}
$$

Then, as is clear, $g_{0}$ is the only one element of $G$ which has fixed points on the ambient space $E_{1} \times E_{2} \times E_{3}$. Hence 


$$
\bar{G} \cong(Z / 2 Z) \oplus(Z / 2 Z), \quad Y_{c}=\bar{Y}_{c} / \bar{G}
$$

where $\bar{G}$ has no fixed points on $\bar{Y}_{c}$. Let

$$
\varphi_{1}: \tilde{X}_{c} \rightarrow \bar{Y}_{c} \text { and } \varphi_{2}: \bar{Y}_{c} \rightarrow Y_{c}
$$

be the projections. Since

\{fixed points of $g_{0}$ on $\left.E_{1} \times E_{2} \times E_{3}\right\}=\left\{2\right.$-division points on $\left.E_{1} \times E_{2} \times E_{3}\right\}$, we obtain

$$
\begin{aligned}
\text { \{fixed points of } \left.g_{0} \text { on } X_{c}\right\} \\
\quad=\left\{2 \text {-division points on } E_{1} \times E_{2} \times E_{3}\right\} \cap X_{c} \\
\quad=\left\{\text { singular points on } X_{c}\right\} .
\end{aligned}
$$

Let $s_{1}, s_{2}, \cdots, s_{n_{c}}$ be all of the singular points on $X_{c}$ and

$$
C_{i}=\imath^{-1}\left(s_{i}\right) \quad i=1,2, \cdots, n_{c}
$$

where $\imath: \tilde{X}_{c} \rightarrow X_{c}$ is the minimal resolution of all singular points on $X_{c}$. Then $C_{i}$ 's are non-singular rational curves with

$$
\left(C_{i}\right)^{2}=-2, \quad C_{i} \cdot C_{j}=0 \quad(i \neq j) .
$$

By considering $g_{0}$ in local coordinates, we obtain

$$
\left\{\text { fixed points of } g_{0} \text { on } \tilde{X}_{c}\right\}=\bigcup_{i=1}^{n_{c}} C_{i} .
$$

Therefore $\bar{Y}_{c}$ is non-singular, the projection

$$
\varphi_{2}: \bar{Y}_{c} \rightarrow Y_{c}
$$

is an unbranched 4-fold covering surface and the projection

$$
\varphi_{1}: \tilde{X}_{c} \rightarrow \bar{Y}_{c}
$$

is a branched double covering surface along a non-singular branch locus $\Gamma=\bigcup_{i=1}^{n_{c}} \bar{C}_{i}$ $\left(\subset \bar{Y}_{c}\right)$ where $\bar{C}_{i}=\varphi_{1}\left(C_{i}\right)^{\prime}$ s are non-singular rational curves with $\left(\bar{C}_{i}\right)^{2}=-4$ and $K_{Y_{c}} \cdot \bar{C}_{i}=2$.

By Lemma 2-1,

$$
\begin{gathered}
K_{\tilde{X}_{c}}^{2}=2 K_{P_{c}}^{2}+2(2-1) K_{Y_{c}} \cdot \Gamma+\frac{(2-1)^{2}}{2} \Gamma^{2}, \\
e\left(\tilde{X}_{c}\right)=2 e\left(\bar{Y}_{c}\right)-(2-1) e(\Gamma)
\end{gathered}
$$

where $K_{\tilde{X}_{c}}^{2}=e\left(\tilde{X}_{c}\right)=48, K_{Y_{c}} \cdot \Gamma=\sum_{i=1}^{n_{c}} K_{Y_{c}} \cdot \bar{C}_{i}=2 n_{c}, \Gamma^{2}=\sum_{i=1}^{n_{c}} \bar{C}_{i}^{2}=-4 n_{c}$ and $e(\Gamma)=$ $\sum_{i=1}^{n_{c}} e\left(\bar{C}_{i}\right)=2 n_{c}$. Therefore 


$$
\begin{gathered}
48=K_{\tilde{X}_{c}}^{2}=2 K_{\bar{Y}_{c}}^{2}+2 \cdot 1 \cdot 2 n_{c}+\frac{1}{2}\left(-4 n_{c}\right)=2 K_{\bar{Y}_{c}}^{2}+2 n_{c}, \\
48=e\left(\tilde{X}_{c}\right)=2 e\left(\bar{Y}_{c}\right)-2 n_{c} .
\end{gathered}
$$

Hence

$$
K_{Y_{c}}^{2}=24-n_{c}, \quad e\left(\bar{Y}_{c}\right)=24+n_{c} .
$$

Since $\varphi_{2}: \bar{Y}_{c} \rightarrow Y_{c}$ is an unbranched and 4-fold covering, we arrive at

$$
K_{Y_{c}}^{2}=\frac{K_{Y_{c}}^{2}}{4}=6-\frac{n_{c}}{4}, \quad e\left(Y_{c}\right)=\frac{e\left(\bar{Y}_{c}\right)}{4}=6+\frac{n_{c}}{4} .
$$

From (ii) of Proposition 3-2, it follows that

$$
H^{0}\left(Y_{c}, \Omega^{1}\right) \cong H^{0}\left(\tilde{X}_{c}, \Omega^{1}\right)^{G} \cong H^{0}\left(E_{1} \times E_{2} \times E_{3}, \Omega^{1}\right)^{G}
$$

where $H^{0}(,)^{G}$ is the subspace of the elements invariant under the action of $G$. Since $d z_{1}, d z_{2}$ and $d z_{3}$ form a system of basis of holomorphic 1-forms on $E_{1} \times E_{2} \times E_{3}$ and the group $G$ contains

$$
g_{0}:\left(z_{1}, z_{2}, z_{3}\right) \rightarrow\left(-z_{1},-z_{2},-z_{3}\right)
$$

we obtain

$$
H^{0}\left(Y_{c}, \Omega^{1}\right) \cong H^{0}\left(E_{1} \times E_{2} \times E_{3}, \Omega^{1}\right)^{G}=0
$$

Hence

$$
q\left(Y_{c}\right)=\operatorname{dim} H^{1}\left(Y_{c}, \mathcal{O}_{Y_{c}}\right)=\operatorname{dim} H^{0}\left(Y_{c}, \Omega^{1}\right)=0 .
$$

By the aboves and Noether's formula (Lemma 2-1), we obtain

$$
\begin{gathered}
p_{g}\left(Y_{c}\right)-0+1=p_{g}\left(Y_{c}\right)-q\left(Y_{c}\right)+1=\frac{K_{Y_{c}}^{2}+e\left(Y_{c}\right)}{12} \\
=\frac{6-n_{c} / 4+6+n_{c} / 4}{12}=1, \\
p_{g}\left(Y_{c}\right)=0 .
\end{gathered}
$$

By considering more precisely the zeros of the 2-canonical forms $\left(\imath * d z_{i} \wedge \imath^{*} d z_{j}\right)^{2}$ $(i \neq j)$ on $\bar{Y}_{c}$, we can express explicitly the 2-canonical divisor $2 K_{Y_{c}}$ :

$$
2 K_{Y_{c}}=2\left(E_{1}+E_{2}+F_{1}+F_{2}\right)
$$

where $E_{1}$ and $E_{2}$ are non-singular elliptic curves with $E_{i}^{2}=-1$ and $F_{1}$ and $F_{2}$ are as follows according as the values of $n_{c}$ :

(Case 0) In case $n_{c}=0$

$F_{1}$ and $F_{2}$ are non-singular curves with $g\left(F_{i}\right)=2$ and $F_{i}^{2}=0$. 
(Case 1) In case $n_{c}=4$

$F_{1}$ is a non-singular curve with $g\left(F_{1}\right)=2$ and $F_{1}^{2}=0$,

$F_{2}$ is a non-singular elliptic curve with $F_{2}^{2}=-1$.

(Case 2) In case $n_{c}=8$

(2-1) $\quad F_{1}$ and $F_{2}$ are non-singular elliptic curves with $F_{i}^{2}=-1$, or

(2-2) $F_{1}$ is a non-singular curve with $g\left(F_{1}\right)=2$ and $F_{1}^{2}=0$, $F_{2}$ is a non-singular rational curve with $F_{2}^{2}=-2$.

(Case 3) In case $n_{c}=12$

$F_{1}$ is a non-singular elliptic curve with $F_{1}^{2}=-1$,

$F_{2}$ is a non-singular rational curve with $F_{2}^{2}=-2$.

(Case 4) In case $n_{c}=16$

$F_{1}$ and $F_{2}$ are non-singular rational curves with $F_{i}^{2}=-2$.

Hence $2 K_{\mathbf{Y}_{c}}$ is effective and contains no exceptional curves of the first kind. In particular $Y_{c}$ is a minimal surface of general type.

End of The OUtline of THe ProOf.

In the following, we shall give some remarks on our examples. For details on these remarks, we refer to $\left[I_{2}\right]$.

REMARK 1. The configuration of $2 K_{Y_{c}}$ is as follows:

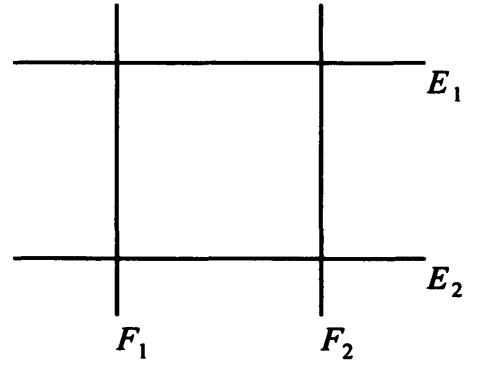

(Case 0)

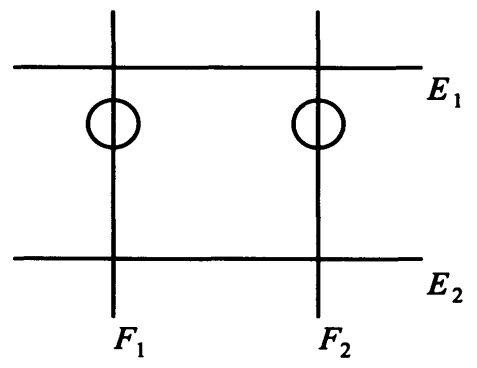

(Case 2-1)

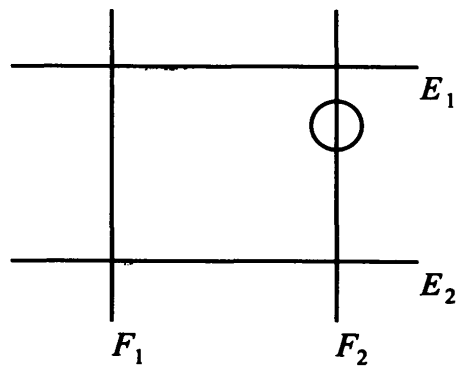

(Case 1)

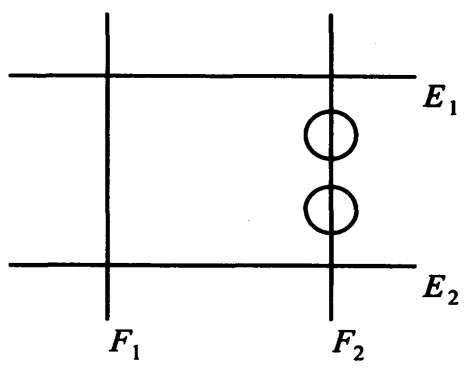

(Case 2-2) 


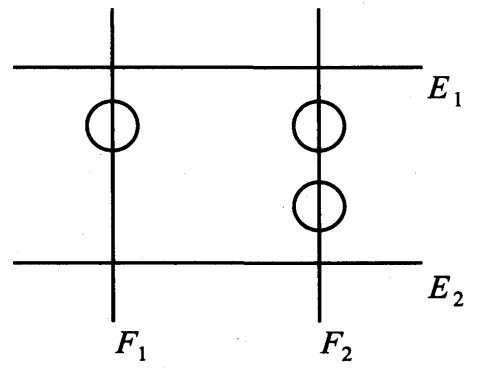

(Case 3)

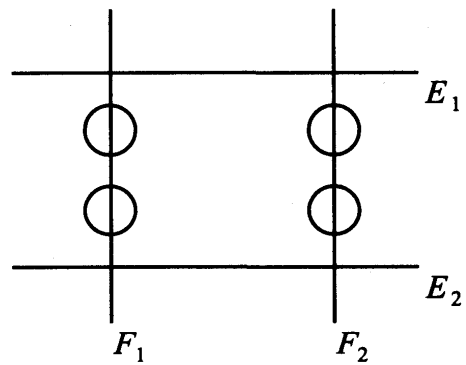

(Case 4)

$\bigcirc$ 's are non-singular rational curves with $(\cdot)^{2}=-4$.

REMARK 2. The fundamental group $\pi_{1}\left(Y_{c}\right)$ and the homology group $H_{1}\left(Y_{c}, Z\right)$ of $Y_{c}$ are as follows. Let $(Z / 2 Z)^{m}$ be the direct sum of $m$ copies of $Z / 2 Z$ and let

$$
H=((Z / 2 Z) *(Z / 2 Z)) /\left\langle\left(\gamma_{1} \gamma_{2} \gamma_{1}^{-1} \gamma_{2}^{-1}\right)^{2}\right\rangle
$$

where $*$ denotes the free product of two groups $Z / 2 Z=\left\langle\gamma_{1}\right\rangle$ and $Z / 2 Z=\left\langle\gamma_{2}\right\rangle$. Then we have the following table:

\begin{tabular}{|r|c|c|c|}
\hline$n_{c}$ & $K_{Y_{c}}^{2}$ & $\pi_{1}\left(Y_{c}\right)$ & $H_{1}\left(Y_{c}, Z\right)$ \\
\hline 0 & 6 & $0 \rightarrow Z^{6} \rightarrow \pi_{1}\left(Y_{c}\right) \rightarrow G \rightarrow 0$ & $(Z / 2 Z)^{6}$ \\
4 & 5 & $H \times(Z / 2 Z)^{3}$ & $(Z / 2 Z)^{5}$ \\
8 & 4 & $H \times(Z / 2 Z)^{2}$ & $(Z / 2 Z)^{4}$ \\
12 & 3 & $H \times(Z / 2 Z)$ & $(Z / 2 Z)^{3}$ \\
16 & 2 & $H$ & $(Z / 2 Z)^{2}$ \\
\hline
\end{tabular}

REMARK 3. In case $\tau_{1}=\tau_{2}=\tau_{3}$ (namely $E_{1}=E_{2}=E_{3}$ ), $E_{1} \times E_{2} \times E_{3}$ admits the cyclic permutation of the components of the coordinates as an automorphism:

$$
\sigma:\left(z_{1}, z_{2}, z_{3}\right) \rightarrow\left(z_{3}, z_{1}, z_{2}\right) .
$$

By the definition of $X_{c}$, the subvariety $X_{c}$ is invariant under the action of $\sigma$. Hence $\sigma$ induces an automorphism

$$
\sigma: \tilde{X}_{c} \rightarrow \tilde{X}_{c}
$$

Since $\sigma^{-1} G \sigma=G, \sigma$ induces an automorphism

$$
\sigma: Y_{c}=\tilde{X}_{c} / G \rightarrow Y_{c}=\tilde{X}_{c} / G
$$

such that $\sigma^{3}=1$. $\sigma$ has isolated fixed points on $Y_{c}$ which are described locally in the following two types: 


$$
\begin{array}{ll}
\text { type 1: } & \sigma:\left(\zeta_{1}, \zeta_{2}\right) \rightarrow\left(\omega \zeta_{1}, \omega^{2} \zeta_{2}\right), \\
\text { type 2: } & \sigma:\left(\zeta_{1}, \zeta_{2}\right) \rightarrow\left(\omega \zeta_{1}, \omega \zeta_{2}\right)
\end{array}
$$

where $\omega^{3}=1$ and $\omega \neq 1$. The number of the fixed points is as follows:

\begin{tabular}{|c|c|c|c|}
\hline$n_{c}$ & number of type 1 & number of type 2 & total \\
\hline 0 & 3 & 0 & 3 \\
4 & 2 & 2 & 4 \\
8 & 1 & 4 & 5 \\
12 & 3 & 0 & 3 \\
16 & 2 & 2 & 4 \\
\hline
\end{tabular}

Let $\rho: Z_{c} \rightarrow Y_{c} \mid\langle\sigma\rangle$ be the minimal resolution of $Y_{c} /\langle\sigma\rangle$. Then for each singular point $s \in Y_{c} /\langle\sigma\rangle, \rho^{-1}(s)$ is as follows:

$$
\text { type 1: } \quad \rho^{-1}(s)=C_{1} \cup C_{2}
$$

where $C_{1}$ and $C_{2}$ are non-singular rational curves with $C_{1} \cdot C_{2}=1$ and $\left(C_{1}\right)^{2}=\left(C_{2}\right)^{2}=-2$.

$$
\text { type 2: } \quad \rho^{-1}(s)=C
$$

where $C$ is a non-singular rational curve with $(C)^{2}=-3$. From these we can derive some results on $Z_{c}: \quad Z_{c}$ is a minimal surface with $p_{g}\left(Z_{c}\right)=q\left(Z_{c}\right)=0$ and

\begin{tabular}{|r|c|l|c|}
\hline$n_{c}$ & $K_{Z_{c}}^{2}$ & structure & $\pi_{1}\left(Z_{c}\right)$ \\
\hline 0 & 2 & general & $(Z / 2 Z) \oplus(Z / 2 Z)$ \\
4 & 1 & general & $Z / 2 Z$ \\
8 & 0 & elliptic & 0 \\
12 & 1 & general & $Z / 2 Z$ \\
16 & 0 & elliptic & 0 \\
\hline
\end{tabular}

REMARK 4. In case $c=b_{1} \cdot b_{2} \cdot b_{3}$ where $b_{i}=\wp_{i}\left(\tau_{i} / 4\right)$, the possible values of $n_{c}$ are 0 and 8 and, by Lemma 3-2, $X_{c}$ is invariant under the action of

$$
h:\left(z_{1}, z_{2}, z_{3}\right) \rightarrow\left(z_{1}+\tau_{1} / 2, z_{2}+\tau_{2} / 2, z_{3}+\tau_{3} / 2\right) .
$$

Since $h$ and each element of $G$ are commutative to each other, $h$ induces an automorphism

$$
h: Y_{c}=\tilde{X}_{c} / G \rightarrow Y_{c}=\tilde{X}_{c} / G
$$


such that $h^{2}=1$. $h$ has four isolated fixed points on $Y_{c}$. Let $W$ be the minimal resolution of $Y_{c} /\langle h\rangle$. Then $W$ is a minimal surface with $p_{g}(W)=q(W)=0$ and

\begin{tabular}{|c|c|c|c|}
\hline$n_{c}$ & $K_{W}^{2}$ & structure & $\pi_{1}(W)$ \\
\hline 0 & 3 & general & $H \times(Z / 2 Z)^{3}$ \\
8 & 2 & general & $H \times(Z / 2 Z)^{2}$ \\
\hline
\end{tabular}

REMARK 5. In case $c=b_{1} \cdot b_{2} \cdot b_{3}$ where $b_{i}=\wp_{i}\left(\tau_{i} / 4\right)$ and, moreover, $b_{1}=b_{2}=b_{3}$. Then $X_{c}$ is invariant under the action of $\sigma$ and $h$. Let $V$ be the minimal resolution of $X_{c} \mid\langle\sigma, h\rangle$. Then $V$ is a minimal surface with $p_{g}(V)=q(V)=0$ and

\begin{tabular}{|c|c|c|c|}
\hline$n_{c}$ & $K_{V}^{2}$ & structure & $\pi_{1}(V)$ \\
\hline 0 & 1 & general & $Z / 2 Z$ \\
8 & 0 & elliptic & 0 \\
\hline
\end{tabular}

REMARK 6. In a similar way, we can construct examples of surfaces $S$ of general type with $p_{g}(S)=0$ and $K_{S}^{2}=7$ and 8 .

[Surfaces with $K_{S}^{2}=7$ ] We take four elliptic curves $E_{i}=C /\left\langle 1, \tau_{i}\right\rangle, i=1,2,3,4$. Let $\left(z_{1}, z_{2}, z_{3}, z_{4}\right)$ be the coordinates of the product $E_{1} \times E_{2} \times E_{3} \times E_{4}$ and

$$
\wp_{i}\left(z_{i}\right)=\wp\left(z_{i}, \tau_{i}\right), \quad a_{i}=\wp_{i}\left(\tau_{i} / 2\right), \quad b_{i}=\wp_{i}\left(\tau_{i} / 4\right) \quad\left(b_{i}^{2}=a_{i}\right)
$$

for $i=1,2,3,4$. We define the subvariety $X$ of $E_{1} \times E_{2} \times E_{3} \times E_{4}$ by

$$
\begin{aligned}
X=\left\{\left(z_{1}, z_{2}, z_{3}, z_{4}\right) \in E_{1} \times E_{2} \times E_{3} \times E_{4} \mid\right. & \\
& \left.\wp_{1}\left(z_{1}\right) \wp_{2}\left(z_{2}\right) \wp_{3}\left(z_{3}\right)=b_{1} \cdot b_{2} \cdot b_{3}, \wp_{3}\left(z_{3}\right) \wp_{4}\left(z_{4}\right)=b_{3} \cdot b_{4}\right\} .
\end{aligned}
$$

We consider several conditions on $a_{i}$ 's:

(C0) $\quad a_{3} a_{4} \neq 1$ and $a_{3} \neq a_{4}$,

(C1-0) $\quad a_{1} \cdot a_{2} \cdot a_{3}=1$,

(C1-i) $\quad a_{i}=a_{j} \cdot a_{k}$ where $(i, j, k)$ is a permutation of $(1,2,3)$,

(C2-0) $\quad a_{1} \cdot a_{2} \cdot a_{4}=1$,

(C2-i) $\quad a_{i}=a_{j} \cdot a_{k}$ where $(i, j, k)$ is a permutation of $(1,2,4)$.

Then we know

(1) $X$ is irreducible if and only if $a_{i}$ 's satisfy (C0).

(2) Under the condition ( $\mathrm{C} 0$ ): 
(2-0) $X$ is non-singular if and only if $a_{i}$ 's satisfy neither (C1)'s nor (C2)'s.

(2-1) If $a_{i}$ 's satisfy one of (C1)'s but none of (C2)'s, or, satisfy one of (C2)'s but none of (C1)'s, then $X$ has 16 ordinary double points and is non-singular elsewhere.

(2-2) If $a_{i}$ 's satisfy one of (C1)'s and one of (C2)'s both, then $X$ has 32 ordinary double points and non-singular elsewhere.

(2-3) Any two of (C1)'s cannot hold simultaneously and any two of (C2)'s also.

Now we assume the condition (C0). Let $n$ be the number of the singular points on $X$. Then the subvariety $X$ is irreducible and non-singular outside exactly $n$ ordinary double points where $n=0,16$ or 32 . Let $\imath: \tilde{X} \rightarrow X$ be the minimal resolution of $X$. We consider the following automorphisms of $E_{1} \times E_{2} \times E_{3} \times E_{4}$ :

$$
\begin{aligned}
& g_{1}:\left(z_{1}, z_{2}, z_{3}, z_{4}\right) \rightarrow\left(-z_{1}+\frac{1}{2}, z_{2}+\frac{1}{2}, z_{3}, z_{4}\right), \\
& g_{2}:\left(z_{1}, z_{2}, z_{3}, z_{4}\right) \rightarrow\left(z_{1},-z_{2}+\frac{1}{2}, z_{3}+\frac{1}{2},-z_{4}+\frac{1}{2}\right), \\
& g_{3}:\left(z_{1}, z_{2}, z_{3}, z_{4}\right) \rightarrow\left(z_{1}+\frac{1}{2}, z_{2},-z_{3}+\frac{1}{2},-z_{4}+\frac{1}{2}\right), \\
& g_{4}:\left(z_{1}, z_{2}, z_{3}, z_{4}\right) \rightarrow\left(z_{1}, z_{2},-z_{3},-z_{4}\right), \\
& g_{5}:\left(z_{1}, z_{2}, z_{3}, z_{4}\right) \rightarrow\left(z_{1}+\tau_{1} / 2, z_{2}+\tau_{2} / 2, z_{3}+\tau_{3} / 2, z_{4}+\tau_{4} / 2\right) .
\end{aligned}
$$

Let $G$ be the group generated by $g_{1}, g_{2}, g_{3}, g_{4}$ and $g_{5}$ :

$$
G=\left\langle g_{1}, g_{2}, g_{3}, g_{4}, g_{5}\right\rangle\left(\cong(Z / 2 Z)^{5}\right) \text {. }
$$

Then $X$ is invariant under the action of $G . G$ operates on $X$ and, hence, naturally on the minimal resolution $\tilde{X}$ of $X$. Let $S$ be the quotient surface of $\tilde{X}$ by $G$. Then we have the following results:

$S$ is a non-singular minimal surface of general type with

$$
p_{g}(S)=q(S)=0, \quad K_{S}^{2}=7-n / 16, \quad e(S)=5+n / 16
$$

where $n=$ the number of the singular points on $X=0,16$ or 32 . Hence

$$
K_{S}^{2}=7,6 \text { or } 5, \quad e(S)=5,6 \text { or } 7
$$

according as $n=0,16$ or 32 .

[Surfaces with $K_{S}^{2}=8$ ] Under the same circumstances, we define the subvariety $X$ of $E_{1} \times E_{2} \times E_{3} \times E_{4}$ by

$$
\begin{aligned}
& X=\left\{\left(z_{1}, z_{2}, z_{3}, z_{4}\right) \in E_{1} \times E_{2} \times E_{3} \times E_{4} \mid\right. \\
&\left.\wp_{1}\left(z_{1}\right) \wp_{2}\left(z_{2}\right)=b_{1} \cdot b_{2}, \wp_{3}\left(z_{3}\right) \wp_{4}\left(z_{4}\right)=b_{3} \cdot b_{4}\right\} .
\end{aligned}
$$

We assume 


$$
\begin{array}{ll}
a_{1} \cdot a_{2} \neq 1, & a_{1} \neq a_{2}, \\
a_{3} \cdot a_{4} \neq 1, & a_{3} \neq a_{4} .
\end{array}
$$

Then $X$ is a product of two irreducible and non-singular algebraic curves. We consider the following automorphisms of $E_{1} \times E_{2} \times E_{3} \times E_{4}$ :

$$
\begin{aligned}
& g_{1}:\left(z_{1}, z_{2}, z_{3}, z_{4}\right) \rightarrow\left(-z_{1}+\frac{1}{2}, z_{2}+\frac{1}{2},-z_{3},-z_{4}\right), \\
& g_{2}:\left(z_{1}, z_{2}, z_{3}, z_{4}\right) \rightarrow\left(-z_{1}+\tau_{1} / 2,-z_{2}+\tau_{2} / 2, z_{3}+\tau_{3} / 2, z_{4}+\tau_{4} / 2\right), \\
& g_{3}:\left(z_{1}, z_{2}, z_{3}, z_{4}\right) \rightarrow\left(z_{1}+\tau_{1} / 2, z_{2}+\tau_{2} / 2,-z_{3}+\frac{1}{2},-z_{4}+\frac{1}{2}\right), \\
& g_{4}:\left(z_{1}, z_{2}, z_{3}, z_{4}\right) \rightarrow\left(-z_{1}+\frac{1}{2},-z_{2}+\frac{1}{2},-z_{3}+\tau_{3} / 2, z_{4}+\tau_{4} / 2\right) .
\end{aligned}
$$

Let $G$ be the group generated by $g_{1}, g_{2}, g_{3}$ and $g_{4}$ :

$$
G=\left\langle g_{1}, g_{2}, g_{3}, g_{4}\right\rangle\left(\cong(Z / 2 Z)^{4}\right) \text {. }
$$

Then $X$ is invariant under the action of $G$. $G$ has no fixed points on $X$. Let $S$ be the quotient surface of $X$ by $G$. Then we have the following results:

$S$ is a non-singular minimal surface of general type with

$$
p_{g}(S)=q(S)=0, \quad K_{S}^{2}=8, \quad e(S)=4 .
$$

\section{References}

[H] F. HiRzebruch, Automorphe Formen und der Satz von Riemann-Roch, Symp. Int. Top. Alg., Univ. Mexico (1958), 129-144.

[ $\left.\mathrm{I}_{1}\right]$ M. INOUE, Miscellaneous examples (I) Some surfaces of general type with positive indices, Aoyama J. Gen. Ed. 33 (1992), 145-173.

[I $\left.\mathrm{I}_{2}\right]$ M. INOUE, Forthcoming paper on some surfaces of general type with $p_{g}=0$, to appear in Aoyama $\mathrm{J}$. Gen. Ed.

[K] K. KodAIRA, A certain type of irregular algebraic surfaces, J. Analyse Math. 19 (1967), $207-215$.

[M] Y. MiYaOKA, Tricanonical maps of numerical Godeaux surfaces, Invent. Math. 34 (1976), 99-111.

[S] T. SHIOdA, On elliptic modular surfaces, J. Math. Soc. Japan 24 (1972), 20-59.

[V] A. VAN DE Ven, On the Chern numbers of certain complex and almost complex manifolds, Proc. Nat. Acad. Sci. U.S.A. 55 (1966), 1624-1627.

\section{Present Address:}

Department of Mathematics, Aoyamagakuin University, Chitosedai, Setagaya-ku, Tokyo, 157 Japan. 